\title{
I can not understand!
}

The United States Preventative Services Task Force (USPSTF) made a final recommendation on May 21, 2012. The recommendation is against prostate-specific antigen (PSA) based screening for healthy men, asserting that there is "moderate or high certainty that the service has no benefit or that the harms outweigh the benefits," discouraged the use of the test by issuing it a Grade D rating. The D rating applies to men of all ages but does not apply to the use of PSA testing for monitoring patients after a prostate cancer diagnosis or treatment.

It is hard to understand the basis of this recommendation. The reaction of the American urological community was strong; the American Urological Association (AUA) called the USPSTF recommendation as inappropriate and irresponsible.

If we banish PSA testing we would go back 25 years ago when most of patients with prostate cancer had an advanced disease and the possible treatments were only palliative.

Furthermore there are many evidences that early diagnosis brings a benefit to prostate cancer patients in terms of mortality reduction. The European Randomized Study for the Screening of Prostate Cancer update, just presented last month at the AUA meeting, showed a $21 \%$ risk reduction in prostate cancer death rate because of the screening.

At this point every urologist agrees that are many prostate cancer patients who do not need to be diagnosed and treated but this is the reason to deny an early diagnosis to that men who would be saved by a radical treatment?

Interestingly, the Göteborg Trial showed 44 percent relative risk reduction in prostate cancer mortality occurring in men 50-64 years of age after a median of 14 years. In this area many patients are not aggressively treated for prostate cancer, indicating that a good clinical practice can prevent unnecessary treatments (1).

Maybe the reason for the tentative of discouraging PSA testing can be economical. The financial burden of thousands of biopsies, robot assisted or not surgeries, radiotherapy sessions probably has been too heavy for the American Healthcare System. If you link to http://csn.cancer.org/node/184022 the website Cancer Survivor Network of American Cancer Society you will find the statement of a man (number 142) who had been submitted to a robot-assisted radical prostatectomy; he detailed all the steps he went through and said that the cost of everything was $\$ 66,157.30$ USD. In this basis to treat $50 \%$ of newly diagnosed prostate cancer/year in the USA the healthcare system would spend 7 billion USD!

There are still many problems with the treatment of prostate cancer. We still do not have markers with adequate sensitivity and specificity for this cancer; we are probably 
overtreating many patients and we are spending too much in the diagnosis and in the treatment, mainly in new technologies with no clear benefits to the patients. But this is not enough reason to banish the only powerful tool we have for an early diagnosis of prostate cancer and probably save many lives.

\section{REFERENCE}

1. Hugosson J, Carlsson S, Aus G, Bergdahl S, Khatami A, Lodding P, et al.: Mortality results from the Göteborg randomised population-based prostate-cancer screening trial. Lancet Oncol. 2010; 11: 725-32.

Dr. SidNEY GLinA

Editor-In-Chief International Braz J Urol 